\title{
Communication \\ Two New Cyototoxic Cardenolides from the Whole Plants of Adonis multiflora Nishikawa \& Koki Ito
}

\author{
Jae-Woo Jung ${ }^{1}$, Nam-In Baek ${ }^{1}$, Jeon Hwang-Bo ${ }^{2}$, Seung-Su Lee ${ }^{1}$, Ji-Hae Park ${ }^{1}$, \\ Kyeong-Hwa Seo ${ }^{1}$, Jung-Hwa Kwon ${ }^{1}$, Eun-Ji Oh ${ }^{1}$, Dae-Young Lee ${ }^{3}$, In-Sik Chung ${ }^{2}$ \\ and Myun-Ho Bang ${ }^{1, *}$ \\ Received: 4 September 2015; Accepted: 13 November 2015; Published: 23 November 2015 \\ Academic Editor: Derek J. McPhee \\ 1 Graduate School of Biotechnology and Department of Oriental Medicine Biotechnology, \\ Kyung Hee University, Yongin 446-701, Korea; jaewoo4848@naver.com (J.-W.J.); \\ nibaek@khu.ac.kr (N.-I.B.); ldkhhghh@khu.ac.kr (S.-S.L.); wlgo3411@hanmail.net (J.-H.P.); \\ kyeonghwaseo@khu.ac.kr (K.-H.S.); life-kjh@hanmail.net (J.-H.K.); jk3172@nate.com (E.-J.O.) \\ 2 Graduate School of Biotechnology and Department of Genetic Engineering, Kyung Hee University, \\ Yongin 446-701, Korea; hbj3286@khu.ac.kr (J.H.-B.); ischung@khu.ac.kr (I.-S.C.) \\ 3 Department of Herbal Crop Research, National Institute of Horicultural and Herbal Science, RDA, \\ Eumseong 369-873, Korea; dylee0809@korea.kr \\ * Correspondence: bangmh68@khu.ac.kr; Tel.: +82-31-888-6175; Fax: +82-31-888-6173
}

\begin{abstract}
A phytochemical investigation of the whole plants of Adonis multiflora Nishikawa \& Koki Ito. resulted in the isolation and identification of two new cardenolides-adonioside A (1) and adonioside B (6) - as well as four known cardenolides: tupichinolide (2) oleandrine (3), cryptostigmin II (4), and cymarin (5). Their structures were elucidated on the basis of NMR, MS, and IR spectroscopic analyses. Compounds 1, 2, 5, and 6 showed significant cytotoxicity against six human cancer cell lines (HCT-116, HepG2, HeLa, SK-OV-3, and SK-MEL-5, and SK-BR-3).
\end{abstract}

Keywords: Adonis multiflora; adonioside A; adonioside B; cardenolide; cytotoxic activity

\section{Introduction}

Cardenolides, a chemical class within the cardiac glycosides, have a five-membered lactone group in the $\beta$ position at C17 [1]. The mechanisms of these compounds are known to inhibit $\mathrm{Na}^{+} / \mathrm{K}^{+}$-ATPase, activate the cation pump, and increase in intracellular calcium concentration through cellular output of $\mathrm{Na}^{+}$and intake of $\mathrm{K}^{+}$[2]. Because of these biological actions, cardenolides have been used in the treatment of heart failure [3]. In addition, many researchers have suggested that cardenolides may inhibit the growth of cancer cells, and have described them as anticancer agents with fewer side effects [4,5].

Cardiac glycosides were isolated from several plant families of Ranunculaceae, Scrophulariazea, Apocynaceae, and Liliaceae, along with pregnane glycosides [6]. In Korea, the Adonis family is mainly comprised of three species, A. amurensis, A. pseudoamurensis, and A. multiflora based on RAPD analysis [7,8]. Previous phytochemical studies conducted on the roots of A. amurensis, the most well-known Adonis species, have identified several cardenolides: corchoroside A, covallatoxin, cymarin, cymarol, digitoxigenin 3-O- $\beta$-D-cymaroside, k-strophanthin, and k-strophanthin- $\beta$ [9]. However, little has been reported concerning the biological and phytochemical properties of A. multiflora, except a brief report [10]. We have confirmed the presence of cardenolide spots in the TLC of ethanolic extracts from whole plants of A. multiflora based on the UV absorption pattern and the colors produced by spraying with a $10 \% \mathrm{H}_{2} \mathrm{SO}_{4}$ solution and heating. Over the course of investigating cardenolides in whole plants of $A$. multiflora Nishikawa \& Koki Ito, two new 
cardenolides $\mathbf{1}$ and $\mathbf{6}$ were identified and structurally determined, along with four known ones 2-5 (Figure 1). The cardenolides were then evaluated for cytotoxity against six human cancer cell lines (HCT-116, HepG2, HeLa, SK-OV-3, SK-BR-3, and SK-MEL-5).
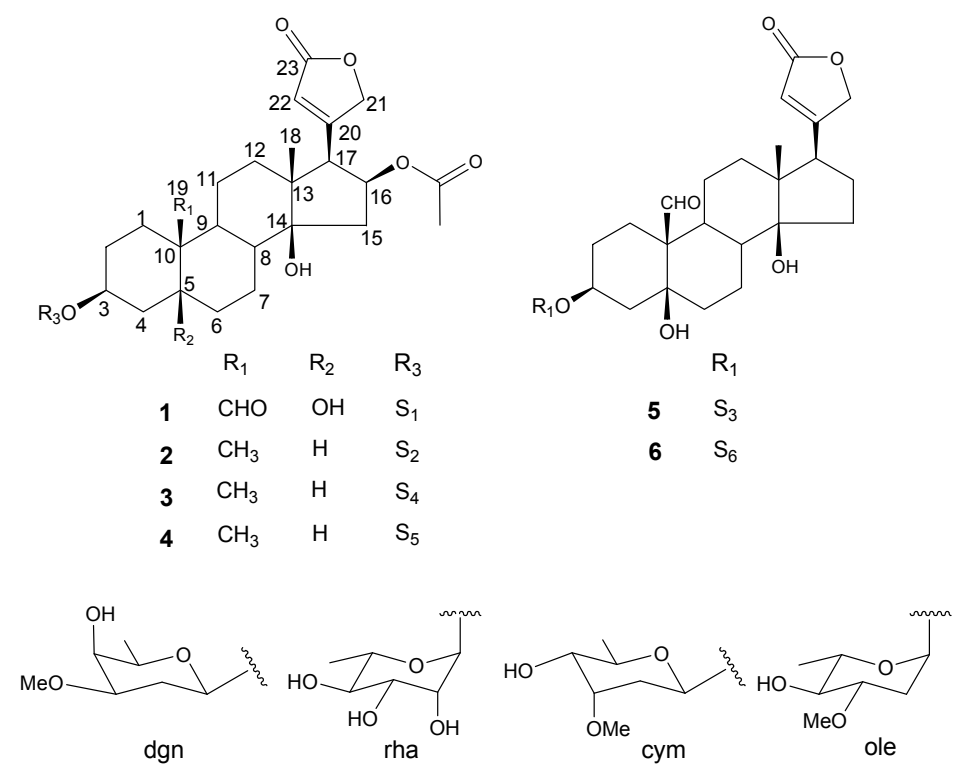

$\mathrm{S}_{1}$

$\mathrm{S}_{2}$
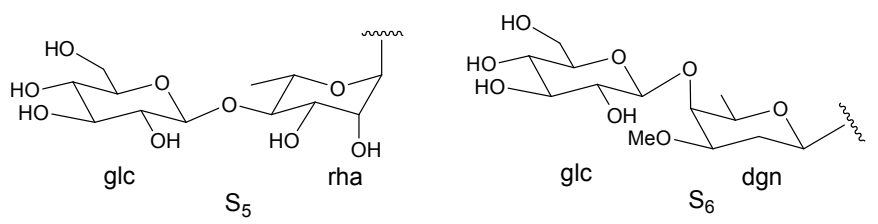

dgn: $\beta$-D-diginopyranose rha: $\alpha$-L-rhamnopyranose glc: $\beta$-D-glucopyranose cym: $\beta$-D-cymaropyranose ole: $\alpha$-L-oleandrose

Figure 1. Compounds 1-6 isolated from the whole plants of Adonis multiflora.

\section{Results and Discussion}

The EtOH extracts were partitioned into $\mathrm{CH}_{2} \mathrm{Cl}_{2}, \mathrm{EtOAc}, \mathrm{BuOH}$, and $\mathrm{H}_{2} \mathrm{O}$ fractions. Repeated $\mathrm{SiO}_{2}$ and ODS column chromatography of the $\mathrm{CH}_{2} \mathrm{Cl}_{2}$ and $\mathrm{BuOH}$ fractions resulted in the identification of two new cardenolides, named adonioside A (1) and adonioside B (6), along with four known cardenolides 2-5. The known compounds were identified as tupichinolide (2), oleandrine (3), cryptostigmin II (4), and cymarin (5) on the basis of spectroscopic analysis and the identities were confirmed by comparing their measured spectroscopic data with those reported in the literature [11-14].

Compound 1 was isolated as a white powder and showed IR absorbance bands representing $\mathrm{OH}$ $\left(3384 \mathrm{~cm}^{-1}\right), \mathrm{CHO}\left(1737 \mathrm{~cm}^{-1}\right)$, and $\mathrm{C}=\mathrm{C}\left(1639 \mathrm{~cm}^{-1}\right)$ groups. The molecular weight was determined to be 606 from the molecular ion peak $m / z 605[\mathrm{M}-\mathrm{H}]^{-}$in the negative FAB-MS spectrum, and a molecular formula of $\mathrm{C}_{32} \mathrm{H}_{46} \mathrm{O}_{11}$ was determined from the high-resolved molecular ion peak $\left([\mathrm{M}-\mathrm{H}]^{-}, m / z\right.$ 605.2971, calc. for $\left.\mathrm{C}_{32} \mathrm{H}_{45} \mathrm{O}_{11}, 605.2962\right)$ in the negative HR-FAB-MS. The ${ }^{1} \mathrm{H}-\mathrm{NMR}$ spectrum (Table 1) exhibited the characteristics of an $\alpha, \beta$-unsaturated- $\gamma$-lactone ring, with signals at $\delta(\mathrm{H}) 5.95(\mathrm{dd}, J=1.6,1.6 \mathrm{~Hz}, \mathrm{H}-22), 4.94\left(\mathrm{dd}, J=18.4,1.6 \mathrm{~Hz}, \mathrm{H}-21_{\mathrm{a}}\right)$, and $4.83(\mathrm{dd}, J=18.4,1.6 \mathrm{~Hz}$, $\left.\mathrm{H}-21_{\mathrm{b}}\right)$ as well as a tertiary methyl signal at $\delta(\mathrm{H}) 0.92(\mathrm{~s}, \mathrm{H}-18)$, a formyl signal at $\delta(\mathrm{H}) 9.92(\mathrm{~s}, \mathrm{H}-19)$, two O-bearing $\mathrm{CH}$ signals at $\delta(\mathrm{H}) 4.19$ (br.s, $\mathrm{H}-3$ ) and 5.41 (ddd, $J=9.6,8.4,8.4 \mathrm{~Hz}, \mathrm{H}-16$ ), and an $\mathrm{AcO}$ signal at $\delta(\mathrm{H}) 1.95(\mathrm{~s}, \mathrm{H}-\mathrm{AcO})$, which suggested the presence of a cardenolide moiety with two 
oxygenated methines and an $\mathrm{AcO}$ group. In addition, a hemiacetal signal at $\delta(\mathrm{H}) 4.50(\mathrm{dd}, J=9.6$, 2.4 Hz, $\left.\mathrm{H}-1^{\prime}\right)$, three O-bearing $\mathrm{CH}$ signals at $\delta(\mathrm{H}) 3.31 \sim 3.67\left(\mathrm{H}-3^{\prime} \sim 5^{\prime}\right)$, an O-bearing $\mathrm{CH}_{3}$ signal at $\delta(\mathrm{H})$

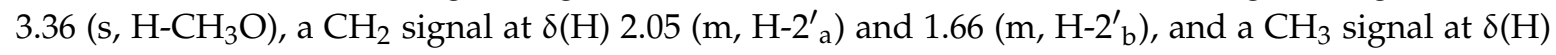
$1.33\left(\mathrm{~d}, J=6.8 \mathrm{~Hz}, \mathrm{H}-6^{\prime}\right)$, indicated that $\mathbf{1}$ was a cardiac monoglycoside with a $\beta$-diginopyranoside.

The ${ }^{13} \mathrm{C}-\mathrm{NMR}$ spectrum showed $32 \mathrm{C}$-atoms signals (Table 1). The aglycone with $\alpha, \beta$-unsaturated- $\gamma$-lactone ring signals observed at $\delta(C) 173.9$ (C-23), 167.3 (C-20), 121.5 (C-22), and 75.5 (C-21), a formyl signal at $\delta(C) 208.1$ (C-19), two O-bearing quaternary signals at $\delta(C) 73.5$ (C-5) and 83.9 (C-14), AcO signals at $\delta(\mathrm{C}) 170.4(\mathrm{C}-\mathrm{OAc})$ and 21.0 (C-OAc), two O-bearing $\mathrm{CH}$ signals at $\delta(\mathrm{C}) 74.1(\mathrm{C}-3)$ and $73.7(\mathrm{C}-16)$, and a tertiary $\mathrm{CH}_{3}$ signal at $\delta(\mathrm{C}) 15.8(\mathrm{C}-18)$ indicated that the aglycone was a cardenolide with four hydroxyls, one formyl, and one $\mathrm{AcO}$ group. The monosaccharide carbon signals, including a hemiacetal signal at $\delta(\mathrm{C}) 98.9\left(\mathrm{C}-1^{\prime}\right)$, three O-bearing $\mathrm{CH}$ signals at $\delta(\mathrm{C}) 77.6\left(\mathrm{C}-3^{\prime}\right)$, $\left.70.7\left(\mathrm{C}-5^{\prime}\right), 66.9 \mathrm{C}-4^{\prime}\right), \mathrm{CH}_{3} \mathrm{O}$ signal at $\delta(\mathrm{C}) 55.8\left(\mathrm{C}-\mathrm{CH}_{3} \mathrm{O}\right)$, a $\mathrm{CH}_{2}$ signal at $\delta(\mathrm{C}) 31.5\left(\mathrm{C}-2^{\prime}\right)$, and a $\mathrm{CH}_{3}$ signal at $\delta(\mathrm{C}) 16.7\left(\mathrm{C}-6^{\prime}\right)$, allowed us to conclude that the sugar was $\beta$-diginopyranose.

Acid hydrolysis of $\mathbf{1}$ and purification of the hydrolysate using column chromatography resulted in a sugar compound, which was identified to be a diginopyranose by direct comparison between its $R_{f}$ values on the $\mathrm{SiO}_{2}$ TLC (0.47 with $\mathrm{CHCl}_{3} / \mathrm{MeOH} 9: 1$, and 0.19 with $\mathrm{CH}_{2} \mathrm{Cl}_{2} / \mathrm{EtOH}$ 9:1) and those of an authentic sample. The specific rotation value of the obtained sugar $\left([\alpha]_{D}^{20}=+56.8, c=0.11, \mathrm{H}_{2} \mathrm{O}\right)$, and the large $J$ value of the anomeric signal at $\delta(\mathrm{H}) 4.50\left(\mathrm{dd}, J=9.6,2.4 \mathrm{~Hz}, \mathrm{H}-\mathrm{1}^{\prime}\right)$ revealed the sugar to be $\beta$-D-diginopyranose. The location of $\beta$-D-diginopyranose, methyl, formyl, hydroxyls, and AcO groups of 1 were determined from the connectivity between the oxygenated methine proton $\delta(\mathrm{H}) 4.50$ $\left(1 \mathrm{H}, \mathrm{dd}, J=9.6,2.4 \mathrm{~Hz}, \mathrm{H}-1^{\prime}\right)$ and O-bearing $\mathrm{CH}$ carbon $\delta(\mathrm{C}) 74.1(\mathrm{C}-3)$, tertiary methyl proton $\delta(\mathrm{H})$ $0.92(\mathrm{~s}, \mathrm{H}-18)$ and quaternary carbon $\delta(\mathrm{C}) 49.8(\mathrm{C}-13)$, formyl proton $\delta(\mathrm{H}) 9.92(\mathrm{~s}, \mathrm{H}-19)$ and quaternary carbon $\delta(\mathrm{C}) 54.3(\mathrm{C}-10)$, tertiary methyl proton $\delta(\mathrm{H}) 0.92(\mathrm{~s}, \mathrm{H}-18)$ and O-bearing quaternary carbon $\delta(\mathrm{C}) 83.9(\mathrm{C}-14)$ and O-bearing methine proton $\delta(\mathrm{H}) 5.41(\mathrm{ddd}, J=9.6,8.4,8.4 \mathrm{~Hz}, \mathrm{H}-16)$ and $\mathrm{AcO}$ carbon $\delta(\mathrm{C}) 170.4(\mathrm{C}-\mathrm{OAc})$ in the HMBC spectrum, respectively. The location of the lactone group was deduced from the connectivity between the methylene protons $\mathrm{H}-15 \delta(\mathrm{H}) 2.59(\mathrm{dd}, J=15.6$, $9.6 \mathrm{~Hz}, \mathrm{H}-15)$, O-bearing methine proton $\delta(\mathrm{H}) 5.41$ (ddd, $J=9.6,8.4,8.4 \mathrm{~Hz}, \mathrm{H}-16$ ) and the methine proton $\delta(\mathrm{H}) 3.15(\mathrm{~d}, J=8.4 \mathrm{~Hz}, \mathrm{H}-17)$ in the COSY spectrum (Figure 2). Taken together, compound 1 was determined to be a $16-\beta$-acetoxystrophanthidin 3-O- $\beta$-D-digonopyranoside, a new cardenolide named adonioside A.

Compound 6 was also isolated as a white powder and showed IR absorbance bands of $\mathrm{OH}$ $\left(3387 \mathrm{~cm}^{-1}\right), \mathrm{CHO}\left(1742 \mathrm{~cm}^{-1}\right)$, and $\mathrm{C}=\mathrm{C}\left(1647 \mathrm{~cm}^{-1}\right)$ groups. The molecular weight was determined to be 710 due to the pseudomolecular ion peak $m / z 733[\mathrm{M}+\mathrm{Na}]^{+}$in the positive FAB-MS spectrum, and the molecular formula of $\mathrm{C}_{36} \mathrm{H}_{54} \mathrm{O}_{14}$ was determined by the high-resolution pseudomolecular ion peak $\left([\mathrm{M}+\mathrm{Na}]^{+}, m / z 733.3511\right.$, calc. for $\mathrm{C}_{36} \mathrm{H}_{54} \mathrm{O}_{14} \mathrm{Na}$, 733.3411) in the positive HR-FAB-MS. The ${ }^{1} \mathrm{H}-\mathrm{NMR}$ spectrum (Table 1) displayed a formyl signal at $\delta(\mathrm{H}) 10.33$ (s, H-19), an olefin $\mathrm{CH}$ signal at $\delta(\mathrm{H}) 6.10(\mathrm{~s}, \mathrm{H}-22)$, O-bearing $\mathrm{CH}_{2}$ signals at $\delta(\mathrm{H}) 5.25\left(\mathrm{~d}, J=18.4 \mathrm{~Hz}, \mathrm{H}-21_{\mathrm{a}}\right)$ and $4.99\left(\mathrm{~d}, J=18.4 \mathrm{~Hz}, \mathrm{H}-21_{\mathrm{b}}\right)$, O-bearing $\mathrm{CH}$ signal at $\delta(\mathrm{H}) 4.64$ (br.s, $\left.\mathrm{H}-3\right)$, and a tertiary $\mathrm{CH}_{3}$ at $\delta(\mathrm{H}) 0.98(\mathrm{~s}, \mathrm{H}-18)$ indicating that 6 has a cardenolide skeleton. Also, two hemiacetal signals at $\delta(\mathrm{H}) 5.07$ (d, $\left.J=7.6 \mathrm{~Hz}, \mathrm{H}-1^{\prime}\right)$ and 4.69 (br.d, $\left.J=9.2 \mathrm{~Hz}, \mathrm{H}-1^{\prime \prime}\right)$ were observed, and their large $J$ values confirmed that the anomer hydroxyls were in $\beta$ form. Two hexoses were determined to be $\beta$-diginopyranosyl-( $1 \rightarrow 4)$ - $\beta$-diginopyranose through comparisons between ${ }^{13} \mathrm{C}$-NMR data and those reported in previously published literature [15]. Acid hydrolysis of 6 and comparison of the specific rotation values of two isolated sugars $\left[\mathbf{6 a}:[\alpha]_{D}^{20}=+55.5\left(c=0.12, \mathrm{H}_{2} \mathrm{O}\right), 6 \mathbf{b}:[\alpha]_{D}^{20}=\right.$ $\left.+49.3\left(c=0.12, \mathrm{H}_{2} \mathrm{O}\right)\right]$ led to the identification of two sugars, D-diginopyranose $\left([\alpha]_{D}^{20}=+59.6\right)$ and D-glucopyranose $\left([\alpha]_{D}^{20}=+52.5\right)[16,17]$. The locations of functional groups were determined by gCOSY and gHMBC experiments (Figure 2). Thus, compound 6 was identified as strophanthidin 3 -O- $\beta$-D-diginopyranosyl-( $(\rightarrow 4)$ - $\beta$-D-glucopyronoside, a new cardenolide named adonioside $B$. 
Table $1 .{ }^{1} \mathrm{H}$ - and ${ }^{13} \mathrm{C}-\mathrm{NMR}$ Data (400 and $100 \mathrm{MHz}$, resp.) of compounds $\mathbf{1}$ and $\mathbf{6}$.

\begin{tabular}{|c|c|c|c|c|}
\hline \multirow[t]{2}{*}{ Position } & \multicolumn{2}{|l|}{$1\left(\mathrm{CD}_{3} \mathrm{OD}\right)$} & \multicolumn{2}{|l|}{$6\left(C_{5} D_{5} N\right)$} \\
\hline & $\delta(\mathrm{H})$ & $\delta(C)$ & $\delta(\mathrm{H})$ & $\delta(C)$ \\
\hline \multirow[t]{2}{*}{1} & $2.05(\mathrm{~m})$ & 23.6 & $2.52(\mathrm{~m})$ & 18.6 \\
\hline & $1.13(\mathrm{~m})$ & & $1.83(\mathrm{~m})$ & \\
\hline \multirow[t]{2}{*}{2} & $2.04(\mathrm{~m})$ & 25.1 & $2.06(\mathrm{~m})$ & 25.5 \\
\hline & $1.42(\mathrm{~m})$ & & $1.64(\mathrm{~m})$ & \\
\hline 3 & 4.19 (br.s) & 74.1 & 4.64 (br.s) & 74.5 \\
\hline \multirow[t]{2}{*}{4} & $1.94(\mathrm{~m})$ & 35.2 & $2.27(\mathrm{~m})$ & 37.0 \\
\hline & $1.62(\mathrm{~m})$ & & $1.81(\mathrm{~m})$ & \\
\hline 5 & & 73.5 & & 73.8 \\
\hline \multirow[t]{2}{*}{6} & $1.93(\mathrm{~m})$ & 35.9 & $2.17(\mathrm{~m})$ & 36.0 \\
\hline & $1.57(\mathrm{~m})$ & & $1.76(\mathrm{~m})$ & \\
\hline \multirow[t]{2}{*}{7} & $1.51(\mathrm{~m})$ & 21.4 & $1.52(\mathrm{~m})$ & 24.8 \\
\hline & $1.43(\mathrm{~m})$ & & $1.32(\mathrm{~m})$ & \\
\hline 8 & $1.96(\mathrm{~m})$ & 41.4 & $2.26(\mathrm{~m})$ & 41.9 \\
\hline 9 & $1.41(\mathrm{~m})$ & 39.0 & $1.64(\mathrm{~m})$ & 39.5 \\
\hline 10 & & 54.3 & & 55.2 \\
\hline \multirow[t]{2}{*}{11} & $2.26($ br.dd, $J=14.8,3.6)$ & 18.1 & $2.43(\mathrm{~m})$ & 22.6 \\
\hline & $1.65(\mathrm{~m})$ & & $1.40(\mathrm{~m})$ & \\
\hline \multirow[t]{2}{*}{12} & $1.55(\mathrm{~m})$ & 39.1 & $1.39(\mathrm{~m})$ & 39.6 \\
\hline & $1.23(\mathrm{~m})$ & & $1.28(\mathrm{~m})$ & \\
\hline 13 & & 49.8 & & 49.8 \\
\hline 14 & & 83.9 & & 84.4 \\
\hline \multirow[t]{2}{*}{15} & $2.59(\mathrm{dd}, J=15.6,9.6)$ & 40.0 & $2.02(\mathrm{~m})$ & 32.2 \\
\hline & $1.74(\mathrm{~m})$ & & $1.79(\mathrm{~m})$ & \\
\hline \multirow[t]{2}{*}{16} & $5.41(\mathrm{ddd}, J=9.6,5.8,2.8)$ & 73.7 & $2.07(\mathrm{~m})$ & 27.2 \\
\hline & & & $1.96(\mathrm{~m})$ & \\
\hline 17 & $3.15(\mathrm{~d}, 8.4)$ & 55.6 & $2.76(\mathrm{~m})$ & 51.1 \\
\hline 18 & $0.92(\mathrm{~s})$ & 15.8 & $0.98(\mathrm{~s})$ & 16.0 \\
\hline 19 & $9.92(\mathrm{~s})$ & 208.1 & $10.33(\mathrm{~s})$ & 208.4 \\
\hline 20 & & 167.3 & & 175.6 \\
\hline \multirow[t]{2}{*}{21} & $4.94(\mathrm{dd}, J=18.4,1.6)$ & 75.5 & $5.25(\mathrm{~d}, J=18.4)$ & 73.7 \\
\hline & $4.83(\mathrm{dd}, J=18.4,1.6)$ & & $4.99(\mathrm{~d}, J=18.4)$ & \\
\hline 22 & $5.95(\mathrm{dd}, J=1.6,1.6)$ & 121.5 & $6.10(\mathrm{~s})$ & 117.8 \\
\hline 23 & & 173.9 & & 174.4 \\
\hline \multirow[t]{2}{*}{$\mathrm{AcO}$} & $1.93(\mathrm{~s})$ & $170.4,21.0$ & & \\
\hline & $\operatorname{Dgn}(a)$ & & Dgn & D-Dig \\
\hline $1^{\prime}$ & $4.50(\mathrm{dd}, J=9.6,2.4)$ & 98.9 & 5.07 (br.d, $J=9.6$ ) & 99.5 \\
\hline \multirow[t]{2}{*}{$2^{\prime}$} & $2.05(\mathrm{~m})$ & 31.5 & $2.15(\mathrm{~m})$ & 32.6 \\
\hline & $1.66(\mathrm{~m})$ & & & \\
\hline $3^{\prime}$ & $3.31(\mathrm{ddd}, J=12.8,5.2,2.0)$ & 77.6 & 3.34 (br.dd, $J=12.0,3.6$ ) & 79.7 \\
\hline $4^{\prime}$ & 3.67 (br.s) & 66.9 & 4.10 (br.s) & 73.9 \\
\hline $5^{\prime}$ & 3.43 (br.q, $J=6.8$ ) & 70.7 & $3.48($ br.q,$J=6.4)$ & 71.1 \\
\hline $6^{\prime}$ & $1.33(\mathrm{~d}, J=6.8)$ & 16.7 & $1.49(\mathrm{~d}, J=6.4)$ & 17.8 \\
\hline \multirow[t]{2}{*}{$\mathrm{MeO}$} & $3.36(\mathrm{~s})$ & 55.8 & $3.28(\mathrm{~s})$ & 56.1 \\
\hline & & & $\mathrm{Glc}^{(\mathrm{b})}$ & \\
\hline $1^{\prime \prime}$ & & & $4.69(\mathrm{~d}, J=7.6)$ & 104.9 \\
\hline $2^{\prime \prime}$ & & & $3.91(\mathrm{dd}, J=8.8,7.6)$ & 75.9 \\
\hline $3^{\prime \prime}$ & & & $4.16(\mathrm{dd}, J=8.8,8.8)$ & 78.5 \\
\hline $4^{\prime \prime}$ & & & $4.10(\mathrm{dd}, J=8.8,8.8)$ & 71.9 \\
\hline $5^{\prime \prime}$ & & & $3.89(\mathrm{~m})$ & 78.3 \\
\hline \multirow{2}{*}{$6^{\prime \prime}$} & & & $4.51(\mathrm{dd}, J=11.2,1.6)$ & 63.1 \\
\hline & & & $4.30(\mathrm{dd}, J=11.2,6.0)$ & \\
\hline
\end{tabular}

(a) $\beta$-D-diginopyranose; ${ }^{(b)} \beta$-D-glucopyranose $\delta$ in ppm, $J$ in Hz. Atom numbering as indicated in Figure 1. 


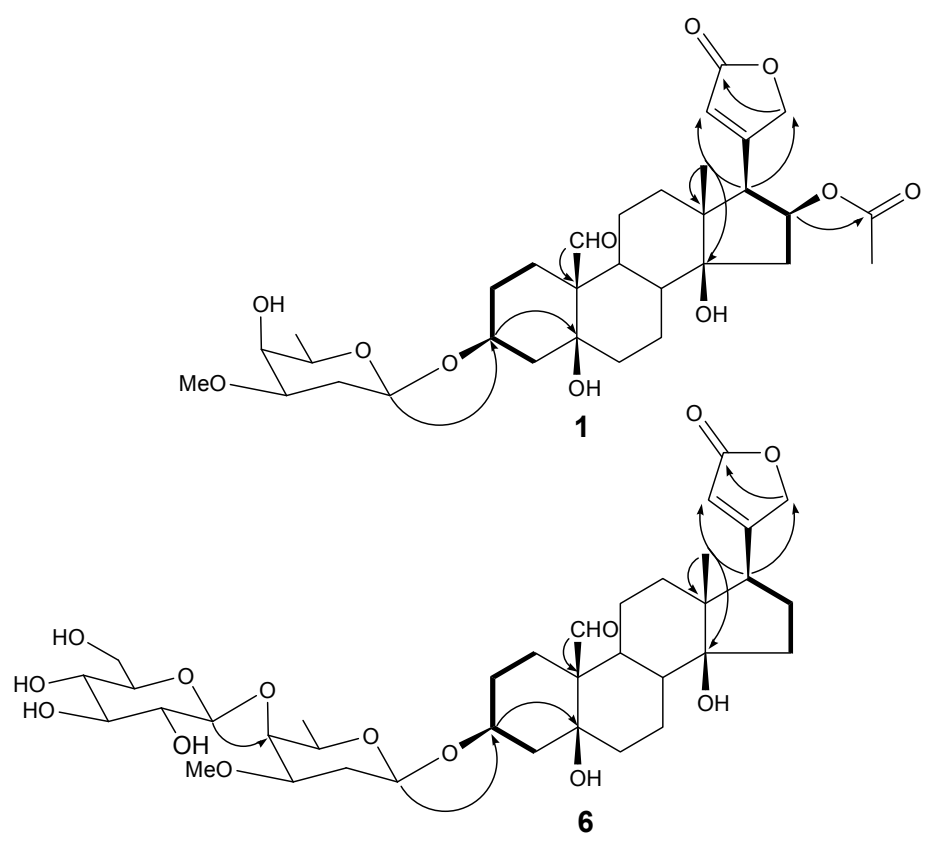

Figure 2. ${ }^{1} \mathrm{H}-{ }^{1} \mathrm{H}-\mathrm{COSY}(-)$ and $\mathrm{gHMBC}(\mathrm{H} \rightarrow \mathrm{C})$ key correlations of compounds $\mathbf{1}$ and $\mathbf{6}$.

All of the isolated cardenolides from A. multiflora were evaluated for cytotoxicity against six human cancer cell lines (HCT-116, HepG2, HeLa, SK-OV-3, SK-BR-3, and SK-MEL-5). As shown in Table 2, compounds 1, 2, 5, and $\mathbf{6}$ showed significant inhibition activity against HCT-116, SK-OV-3, and SK-MEL-5 cell lines with $\mathrm{IC}_{50}$ values ranging from $0.06 \pm 0.02$ to $7.44 \pm 1.98 \mu \mathrm{M}$. Compound 3 showed cytotoxic effects against the HeLa cell line with an $\mathrm{IC}_{50}$ value of $8.85 \pm 0.39 \mu \mathrm{M}$. Compound 4 showed cytotoxicity against the SK-MEL-5 cell line with an $\mathrm{IC}_{50}$ value of $1.99 \pm 0.28 \mu \mathrm{M}$.

Table 2. Cytotoxic activity of compounds $\mathbf{1 - 6}$ against human cancer cell lines $\left(\mathrm{IC}_{50}[\mu \mathrm{M}]^{(\mathrm{a})}\right)$.

\begin{tabular}{ccccccc}
\hline \multirow{2}{*}{ Compound } & \multicolumn{7}{c}{ Cell lines $\left(\right.$ IC $\left._{\mathbf{5 0}}\right) \boldsymbol{\mu M}$} \\
\cline { 2 - 7 } & HCT-116 & HepG2 & HeLa & SK-OV-3 & SK-MEL-5 & SK-BR-3 \\
\hline $\mathbf{1}$ & $4.10 \pm 0.38$ & $14.65 \pm 0.47$ & $38.54 \pm 1.08$ & $2.34 \pm 0.10$ & $3.40 \pm 0.67$ & $38.35 \pm 1.49$ \\
$\mathbf{2}$ & $0.41 \pm 0.13$ & $17.99 \pm 0.61$ & $9.38 \pm 0.15$ & $0.06 \pm 0.02$ & $0.28 \pm 0.06$ & $2.58 \pm 0.23$ \\
$\mathbf{3}$ & $34.99 \pm 1.39$ & $30.12 \pm 1.60$ & $8.85 \pm 0.39$ & $25.38 \pm 0.51$ & $34.17 \pm 1.78$ & $80.38 \pm 1.13$ \\
$\mathbf{4}$ & $24.32 \pm 1.26$ & $26.61 \pm 0.70$ & $23.27 \pm 1.73$ & $41.02 \pm 0.13$ & $1.99 \pm 0.28$ & $23.94 \pm 1.47$ \\
$\mathbf{5}$ & $1.64 \pm 0.13$ & $2.87 \pm 0.77$ & $25.38 \pm 0.15$ & $0.76 \pm 0.15$ & $0.73 \pm 0.14$ & $5.10 \pm 0.87$ \\
$\mathbf{6}$ & $7.44 \pm 1.98$ & $13.71 \pm 0.75$ & $44.71 \pm 0.89$ & $4.63 \pm 0.47$ & $4.98 \pm 0.56$ & $21.30 \pm 1.50$ \\
Doxorubicin & $9.20 \pm 0.90$ & $27.30 \pm 0.50$ & $3.20 \pm 0.30$ & $0.58 \pm 0.08$ & $4.80 \pm 0.23$ & $0.71 \pm 0.05$ \\
\hline \multicolumn{7}{c}{ (a) All data were represented as mean + SD of triplicate experiments. }
\end{tabular}

\section{Experimental Section}

\subsection{General}

Column chromatography (CC): $\mathrm{SiO}_{2}$ (Kieselgel 60, Merck, Darmastdt, Germany) and ODS (LiChroprep RP-18, Merck) resins. TLC: Kieselgel $60 \mathrm{~F}_{254}$ and RP-18 $\mathrm{F}_{254 \mathrm{~S}}$ (Merck) plates; visualization with UV lamp Spectroline Model ENF-240 C/F (Spectronics Corporation, Westbury, NY, USA) and spraying $10 \% \mathrm{H}_{2} \mathrm{SO}_{4}$ soln. in $\mathrm{MeOH}$ and heating. Optical rotations: JASCO P-1010 digital polarimeter (Jasco, Tokyo, Japan). IR spectra: Perkin Elmer Spectrum One FT-IR spectrometer (Perkin Elmer, Beaconsfield, UK). FAB-MS: JEOL JMSAX-700 mass spectrometer (Jeol, Tokyo, Japan). NMR spectra: Varian Unity Inova AS-400 FT-NMR spectrometer (Varian, Palo Alto, CA, USA). 


\subsection{Plant Materials}

A. multiflora Nishikawa \& Koki Ito was supplied from the BMI Corporation (Uiwang, Korea) in January 2014, and was identified by professor Dae-Keun Kim, College of Pharmacy, Woosuk University, Jeonju, Korea. A voucher specimen (KHU2014-0117) has been reserved at the Laboratory of Natural Products Chemistry, Kyung Hee University, Yongin, Korea.

\subsection{Extraction and Isolation}

The whole plants of $A$. multiflora $(1.5 \mathrm{~kg})$ were extracted with $70 \%$ aqueous $\mathrm{EtOH}(30 \mathrm{~L})$ at room temperature for $24 \mathrm{~h}$. The concentrated $\mathrm{EtOH}$ extracts $(106 \mathrm{~g})$ were suspended in $\mathrm{H}_{2} \mathrm{O}(3 \mathrm{~L})$ and then successively extracted with $\mathrm{CH}_{2} \mathrm{Cl}_{2}$ (AAC; $2.6 \mathrm{~g}$ ), AcOEt (AAE; $0.7 \mathrm{~g}$ ), $\mathrm{BuOH}\left(\mathrm{AAB} ; 12 \mathrm{~g}\right.$ ), and $\mathrm{H}_{2} \mathrm{O}$ (AAW; 89.2 g). The AAC (2.6 g) was subjected to $\mathrm{CC}\left[\mathrm{SiO}_{2}(\varphi 4 \times 11 \mathrm{~cm}) ; \mathrm{CH}_{2} \mathrm{Cl}_{2} / \mathrm{MeOH} 18: 1,15: 1\right.$, 7:1, $1.6 \mathrm{~L}$ of each] yielding 16 fractions, AAC-1-AAC-16. Fr. AAC-3 $(200 \mathrm{mg}$, elution volume/total volume $\left(\mathrm{V}_{\mathrm{e}} / \mathrm{V}_{\mathrm{t}}\right)$ 0.03-0.06) was subjected to CC [ODS $(\varphi 3 \times 7 \mathrm{~cm}) ; \mathrm{MeOH} / \mathrm{H}_{2} \mathrm{O} 3: 1,2.4 \mathrm{~L}$, yielding 14 fractions, AAC-3-1-AAC-3-14. Fr. AAC-3-1 (52 mg, $\mathrm{V}_{\mathrm{e}} / \mathrm{V}_{\mathrm{t}}$ 0.00-0.09) was subjected to CC $\left[\mathrm{SiO}_{2}\right.$ $(\varphi 1.5 \times 15 \mathrm{~cm})$; Hexane/AcOEt 1:12, $0.5 \mathrm{~L}$ ], yielding six fractions, AAC-3-1-1-AAC-3-1-6 along with a purified compound 1 [AAC-3-1-2; $12 \mathrm{mg} ; \mathrm{V}_{\mathrm{e}} / \mathrm{V}_{\mathrm{t}}$ 0.46-0.52; TLC (ODS $\mathrm{F}_{254 \mathrm{~S}} ; \mathrm{MeOH} / \mathrm{H}_{2} \mathrm{O}$ 5:2): $R_{f}$ 0.60]. Fr. AAC-4 (130 mg, $\mathrm{V}_{\mathrm{e}} / \mathrm{V}_{\mathrm{t}}$ 0.06-0.08) was subjected to CC [ODS $(\varphi 3 \times 7 \mathrm{~cm}) ; \mathrm{MeOH} / \mathrm{H}_{2} \mathrm{O}$ 4:5, $1.3 \mathrm{~L}$ ], yielding 14 fractions, AAC-4-1-AAC-4-12 along with a purified compound 5 [AAC-4-9; $40 \mathrm{mg} ; \mathrm{V}_{\mathrm{e}} / \mathrm{V}_{\mathrm{t}}$ 0.63-0.81; TLC (ODS $\left.\mathrm{F}_{254 \mathrm{~S}} ; \mathrm{MeOH} / \mathrm{H}_{2} \mathrm{O} 3: 2\right): R_{f}$ 0.45]. Fr. AAC-7 (200 mg, $\mathrm{V}_{\mathrm{e}} / \mathrm{V}_{\mathrm{t}}$ 0.17-0.22) was subjected to CC [ODS $\left.(\varphi 3 \times 5 \mathrm{~cm}) ; \mathrm{MeOH} / \mathrm{H}_{2} \mathrm{O} 3: 1,1.61\right]$, yielding nine fractions, AAC-7-1-AAC-7-9 along with a purified compound 2 [AAC-7-2; $12 \mathrm{mg} ; \mathrm{V}_{\mathrm{e}} / \mathrm{V}_{\mathrm{t}}$ 0.05-0.07; TLC (ODS $\left.\mathrm{F}_{254 \mathrm{~S}} ; \mathrm{MeOH} / \mathrm{H}_{2} \mathrm{O} 4: 1\right): R_{f}$ 0.45]. Fr. AAC-14 (121 mg, $\mathrm{V}_{\mathrm{e}} / \mathrm{V}_{\mathrm{t}}$ 0.62-0.66) was subjected to CC [ODS $(\varphi 3 \times 6 \mathrm{~cm}) ; \mathrm{MeOH} / \mathrm{H}_{2} \mathrm{O} 1: 2,2.7 \mathrm{~L}$, yielding 11 fractions, AAC-14-1-AAC-14-11 along with a purified compound 3 [AAC-14-2; $8 \mathrm{mg} ; \mathrm{V}_{\mathrm{e}} / \mathrm{V}_{\mathrm{t}}$ 0.04-0.19; TLC (ODS F $\left.\mathrm{F}_{2545} ; \mathrm{MeOH} / \mathrm{H}_{2} \mathrm{O} 2: 1\right): R_{f} 0.60$ ], and compound 4 [AAC-14-6; $12 \mathrm{mg} ; \mathrm{V}_{\mathrm{e}} / \mathrm{V}_{\mathrm{t}}$ 0.53-0.69; TLC (ODS F $\left.254 \mathrm{~s} ; \mathrm{MeOH} / \mathrm{H}_{2} \mathrm{O} 2: 1\right): \mathrm{R}_{\mathrm{f}}$ 0.50]. The AAB (12 g) was subjected to $\mathrm{CC}\left[\mathrm{SiO}_{2}(\varphi 7.5 \times 16 \mathrm{~cm}) ; \mathrm{CH}_{2} \mathrm{Cl}_{2} / \mathrm{MeOH} / \mathrm{H}_{2} \mathrm{O}\right.$ 13:3:1, 9:3:1, 7:3:1, 65:35:10, $7 \mathrm{~L}$ of each] yielding 15 fractions, AAB-1-AAC- 15 . Fr. AAC-5 (300 mg, $\left.\mathrm{V}_{\mathrm{e}} / \mathrm{V}_{\mathrm{t}} 0.06-0.10\right)$ was subjected to CC [ODS $\left.(\varphi 2.5 \times 5 \mathrm{~cm}) ; \mathrm{MeOH} / \mathrm{H}_{2} \mathrm{O} 2: 3,1 \mathrm{~L}\right]$, yielding nine fractions, AAB-5-1-AAC-5-9 along with a purified compound 6 [AAB-5-7; $28 \mathrm{mg} ; \mathrm{V}_{\mathrm{e}} / \mathrm{V}_{\mathrm{t}} 0.38-0.71$; TLC $\left(\mathrm{ODS} \mathrm{F}_{254 \mathrm{~S}} ; \mathrm{MeOH} / \mathrm{H}_{2} \mathrm{O}\right.$ 6:5): $\left.R_{f} 0.30\right]$.

\subsection{Spectroscopic Data}

Adonioside $A$ (1). White powder. $[\alpha]_{D}^{20}=+23.9(c=0.5, \mathrm{MeOH})$. IR $\left(\mathrm{CaF}_{2}\right)$ : 3384, 2923, 1737, 1639, 1167, $1077 \mathrm{~cm}^{-1}$. ${ }^{1} \mathrm{H}$ - and ${ }^{13} \mathrm{C}-\mathrm{NMR}$ : Table 1. negative HR-FAB-MS: $605.2971\left([\mathrm{M}-\mathrm{H}]^{-}, \mathrm{C}_{32} \mathrm{H}_{45} \mathrm{O}_{11}\right.$; calc. 605.2962).

Adonioside B (6). White powder. $[\alpha]_{D}^{20}=-94.4\left(c=0.7\right.$, pyridine). IR $\left(\mathrm{CaF}_{2}\right)$ : 3387, 2933, 1742, 1647, 1178, $1097 \mathrm{~cm}^{-1}$. ${ }^{1} \mathrm{H}$ - and ${ }^{13} \mathrm{C}-\mathrm{NMR}$ : Table 1. positive HR-FAB-MS: $733.3511\left([\mathrm{M}+\mathrm{Na}]^{+}\right.$, $\mathrm{C}_{36} \mathrm{H}_{54} \mathrm{O}_{14} \mathrm{Na}$; calc. 733.3414).

\subsection{Acid Hydrolysis of $\mathbf{1}$ and $\mathbf{6}$}

Compound $1(10 \mathrm{mg})$ and compound $6(20 \mathrm{mg})$ were refluxed in $2 \mathrm{~N} \mathrm{HCl}(0.3 \mathrm{~mL})$ at $80{ }^{\circ} \mathrm{C}$ for $5 \mathrm{~h}$, followed by neutralization with $\mathrm{Ag}_{2} \mathrm{CO}_{3}$ in excess and filtered through filter paper. The filtrate of $\mathbf{1}$ was subjected to $\mathrm{CC}$ [ $\left.\mathrm{SiO}_{2}(\varphi 1 \times 10 \mathrm{~cm}) ; \mathrm{CHCl}_{3} / \mathrm{MeOH} 12: 1\right]$ to give fractions of sugar (1a) and aglycone, and that of 6 was subjected to $\mathrm{CC}\left[\mathrm{SiO}_{2}(\varphi 1 \times 10 \mathrm{~cm}) ; \mathrm{CHCl}_{3} / \mathrm{MeOH} 12: 1,1: 1\right]$ to give fractions of sugars $\mathbf{6 a}$, and $\mathbf{6 b}$ and aglycone. The monosaccharides $\mathbf{1 a}, \mathbf{6 a}$, and $\mathbf{6 b}$ in each sugar fraction were identified to be diginose, diginose, and glucose, respectively, by TLC comparison with authentic sugars. The $R_{f}$ values of diginose was 0.37 with $\mathrm{CHCl}_{3} / \mathrm{MeOH} 9: 1$ and 0.47 with $\mathrm{CH}_{2} \mathrm{Cl}_{2} / \mathrm{EtOH}$ 9:1, and that of glucose was 0.30 with $\mathrm{CHCl}_{3} / \mathrm{MeOH} / \mathrm{H}_{2} \mathrm{O}$ 7:3:0.5 [18,19]. 


\subsection{Determination of Absolute Configuration of $\mathbf{1 a}, \mathbf{6} \mathbf{a}$, and $\mathbf{6} \mathbf{b}$}

The sugar fractions, $\mathbf{1 a}(1 \mathrm{mg}), \mathbf{6 a}(1.2 \mathrm{mg})$, and $\mathbf{6 b}(1.2 \mathrm{mg})$, were measured for optical rotation values and compared with those reported in literature. Diginose, $\mathbf{1 a}$ and $\mathbf{6 a}$, were determined to be D-form [1a: $[\alpha]_{D}^{20}=+56.8\left(c=0.11, \mathrm{H}_{2} \mathrm{O}\right), 6 \mathrm{a}:[\alpha]_{D}^{20}=+55.5\left(c=0.12, \mathrm{H}_{2} \mathrm{O}\right)$; D-diginose: $\left.[\alpha]_{D}^{20}=+59.6\right]$. Glucose $6 \mathbf{b}$ was determined to be D-form $\left[\mathbf{6 b}:\left([\alpha]_{D}^{20}=+49.3\left(c=0.12, \mathrm{H}_{2} \mathrm{O}\right)\right.\right.$; D-glucose: $\left.[\alpha]_{D}^{20}=+52.5\right][16,17]$.

\subsection{Cell Culture}

Human hepatoma (HepG2), human cervix adenocarcinoma (HeLa), human ovarian adenocarcinoma (SK-OV-3), human breast adenocarcinoma (SK-BR-3), human colon carcinoma (HCT-116), human melanoma (SK-MEL-5) cells were obtained from the Korean Cell Line Bank (KCLB, Seoul, Korea). HepG2 and HeLa cells were maintained in Dulbecco's modified Eagle's medium (DMEM) supplemented with 10\% $(v / v)$ heat-inactivated fetal bovine serum (FBS) and $1 \%(v / v)$ penicillin-streptomycin in a humidified incubator with $5 \% \mathrm{CO}_{2}$ at $37^{\circ} \mathrm{C}$. SK-OV-3, SK-BR-3, HCT-116, and SK-MEL-5 cells were maintained in RPMI1640 medium containing $10 \%(v / v)$ heat-inactivated FBS and $1 \%(v / v)$ penicillin-streptomycin in a humidified incubator with $5 \% \mathrm{CO}_{2}$ at $37{ }^{\circ} \mathrm{C}$. All cell culture media and reagents were purchased form Thermo Scientific Hyclone (Logan, UT, USA).

\subsection{Cytotoxicity Assay}

The cytotoxicity of cardenolides from A. multiflora was measured by a MTT colorimetric assay. Compounds were dissolved with dimethylsulfoxide (DMSO). The cells were seeded onto 96-well microplates at a density of $1 \times 10^{4}$ cells per well in $100 \mu \mathrm{L}$ of medium each. After incubation at $37^{\circ} \mathrm{C}$ in a humidified incubator for $24 \mathrm{~h}$, the cells were treated with various concentrations $(1,0.1,0.5,1,5$, $10,50,100 \mu \mathrm{M})$ of each compound in serum-free medium for $24 \mathrm{~h}$. After incubation, $50 \mu \mathrm{L}$ of MTT $\left(5 \mathrm{mg} / \mathrm{mL}\right.$ in PBS) was added to each well of the plate. The cells were incubated at $37^{\circ} \mathrm{C}$ for $2 \mathrm{~h}$. After removal from the medium, the cells were treated with $100 \mu \mathrm{L}$ DMSO for 5 min and optical density measured using a microplate reader (BIO-TEK Inc., Winooski, VT, USA) at $550 \mathrm{~nm}$. Cell viability was calculated as a percentage of viable cells in the compound-treated group ws. the control group by the following equation: Cell viability $(\%)=$ [OD (Compound) - OD (Blank)/OD (Control)-OD (Blank)] $\times 100$.

\subsection{Statistical Analysis}

All experiments were performed with triplicate samples and repeated at least three times. The data are presented as means \pm SD and statistical comparisons between groups were performed using 1-way ANOVA followed by Student's $t$-test.

\section{Conclusions}

Two new and four known cardiac glycosides were isolated from the whole plants of Adonis multiflora Nishikawa \& Koki Ito using open column chromatography and were identified based on spectroscopic data analysis, including NMR and FAB-MS. For the determination of absolute configuration, acid hydrolysis was performed. As a result, compound $\mathbf{1}$ and $\mathbf{6}$ were determined to be a 16 - $\beta$-acetoxystrophanthidin 3-O- $\beta$-D-digonopyranoside, named adonioside $\mathrm{A}(\mathbf{1})$ and strophanthidin 3-O- $\beta$-D-diginopyranosyl-( $1 \rightarrow 4)$ - $\beta$-D-glucopyronoside, named adonioside B (6). In addition, the two new compounds 1 and 6 together with the two known compounds 2 and 5 showed significant cytotoxicity against six human cancer cell lines, HCT-116, HepG2, HeLa, SK-OV-3, and SK-MEL-5, and SK-BR-3, but we couldn't establish a consistent structure-activity relationship. Consequently, these four compounds 1, 2, 5 and 6 merit futher in vivo study and on normal cell lines for bioactive selectivity. These findings suggest that $A$. multiflora may have potential be a useful therapeutic natural source for cancer prevention. 
Supplementary Materials: ${ }^{1} \mathrm{H}-\mathrm{NMR}$ and ${ }^{13} \mathrm{C}-\mathrm{NMR}$ spectra of 1 and 6 and HMBC spectrum of 6 are available as supporting data. Supplementary materials can be accessed at: http://www.mdpi.com/1420-3049/20/ $11 / 19722 / s 1$.

Acknowledgments: This study was supported by the grant from Kyung Hee University in 2009 (KHU-20100160).

Author Contributions: M.-H.B., N.-I.B., and I.-S.C. designed research; J.-W.J., J.H.-B., S.-S.L., J.-H.P., K.-H.S., J.-H.K., E.-J.O., and D.-Y.L. performed research and analyzed the data; J.-W.J. wrote the paper. All authors read and approved the final manuscript.

Conflicts of Interest: The authors declare no conflict of interest.

\section{References}

1. Agrawal, A.A.; Petschenka, G.; Bingham, R.A.; Weber, M.G.; Rasmann, S. Toxic cardenolides: Chemical ecology and coevolution of specialized plant-herbivore interactions. New Phytol. 2012, 194, $28-45$. [CrossRef] [PubMed]

2. Rose, A.M.; Valdes, R. Understanding the sodium pump and its relevance to disease. Clin. Chem. 1994, 40, 1674-1685. [PubMed]

3. Prassas, I.; Diamandis, E.P. Novel therapeutic applications of cardiac glycosides. Nat. Rev. Drug Discov. 2008, 7, 926-935. [CrossRef] [PubMed]

4. Dong, W.H.; Mei, W.L.; Zhao, Y.X.; Zeng, Y.B.; Zuo, W.J.; Wang, H.; Li, X.N.; Dai, H.F. Cytotoxic cardenolide glycosides from the seeds of Antiaris toxicaria. Planta Med. 2011, 77, 1730-1734. [CrossRef] [PubMed]

5. Li, Y.; Liu, Y.B.; Yu, S.S.; Chen, X.G.; Wu, X.F.; Ma, S.G.; Qu, J.; Hu, Y.C.; Liu, J.; Lv, H.N. Cytotoxic cardenolides from the stems of Periploca forrestii. Steroids 2012, 77, 375-381. [CrossRef] [PubMed]

6. Kuroda, M.; Kubo, S.; Matsuo, Y.; Atou, T.; Satoh, J.; Fujino, T.; Hayakawa, M.; Mimaki, Y. New cardenolide glycosides from the seeds of Digitalis purpurea and their cytotoxic activity. Biosci. Biotechnol. Biochem. 2013, 6, 1186-1192. [CrossRef] [PubMed]

7. Suh, Y.B.; Lee, J.K.; Lee, S.T.; Lee, C.H.; Yeau, S.H.; Lee, N.S. Molecular evidence for the taxonomic identity of Korean Adonis (Ranunculaceae). J. Plant Res. 2002, 115, 217-223. [CrossRef] [PubMed]

8. Lee, S.T.; Lee, J.H.; Jeong, Y.J.; Seo, Y.B.; Yeau, S.H.; Lee, N.S. A principal components analysis of reproductive characters of Korean Adonis plants (Ranunculaceae). Korean J. Plant Taxon. 2000, 30, 303-313.

9. Ponomarenko, A.A.; Komissarenko, N.F.; Stukkei, K.L. Cardenolides of Adonis amurensis. Chem. Nat. Compd. 1971, 7, 833-834. [CrossRef]

10. Baek, Y.S.; Jung, J.W.; Lee, S.H.; Baek, N.I.; Park, J.H. A new pregnane hexaglycoside from Adonis multiflora. J. Korean Soc. Appl. Biol. Chem. 2015, 58, 895-899. [CrossRef]

11. Pan, Z.H.; Li, Y.; Liu, J.L.; Ning, D.S.; Li, D.P.; Wu, X.D.; Wen, Y.X. A cytotoxic cardenolide and a saponin from the rhizomes of Tupistra chinensis. Fitoterapia 2012, 83, 1489-1493. [CrossRef] [PubMed]

12. Abe, F.; Yamauchi, T.; Minato, K. Presence of cardenolides and ursolic acid from oleander leaves in larvae and frass of Daphnis nerii. Phytochemistry 1996, 42, 45-49. [CrossRef]

13. Kamel, M.S.; Assaf, M.H.; Abe, Y.; Ohtani, K.; Kasai, R.; Yamasaki, K. Cardiac glycosides from Cryptostegia grandiflora. Phytochemistry 2001, 58, 537-542. [CrossRef]

14. Carvalho, J.E.D.; Souccar, C.; Tersariol, I.L.; Torres, L.B.; Lapa, A.J. Pharmacological Properties and Identification of Cardiotonic principles from the indian snuff, Maquira sclerophylla, Ducke. Phytother. Res. 1997, 11, 136-141. [CrossRef]

15. Kuroda, M.; Kubo, S.; Uchida, S.; Sakagami, H.; Mimaki, Y. Amurensiosides A-K, 11 new pregnane glycosides from the roots of Adonis amurensis. Steroids 2010, 75, 83-94. [CrossRef] [PubMed]

16. Tsukamoto, S.; Kaneko, K.; Hayashi, K. A method to Identify the absolute configuration of rhamnose, lyxose, and 2, 6-dideoxy sugars, cymarose, oleandrose, diginose, and digitoxose, using a chiral high-performance liquid chromatography (HPLC) column. Chem. Pharm. Bull. 1989, 37, 637-641. [CrossRef]

17. Perrone, A.; Capasso, A.; Festa, M.; Kemertelidze, E.; Pizza, C.; Skhirtladze, A.; Piacente, S. Antiproliferative steroidal glycosides from digitalis ciliate. Fitoterapia 2012, 83, 554-562. [CrossRef] [PubMed] 
18. Bai, H.; Li, W.; Koike, K. Pregnane glycosides from Cynanchum atratum. Steroids 2008, 73, 96-103. [CrossRef] [PubMed]

19. Gan, H.; Xiang, W.J.; Ma, L.; Hu, L.H. Six New C21 Steroidal glycosides from Cynanchum bungei Decne. Helvetica Chim. Acta 2008, 91, 2222-2224. [CrossRef]

Sample Availability: Samples of the compounds 1-6 are available from the authors.

(C) 2015 by the authors; licensee MDPI, Basel, Switzerland. This article is an open access article distributed under the terms and conditions of the Creative Commons by Attribution (CC-BY) license (http://creativecommons.org/licenses/by/4.0/). 